ISAHP 1999, Kobe, Japan, August 12-14,1999

\title{
INTER-VIEWPOINT STRESS METHODS AND GROUP DECISION MAKING STRESS METHOD
}

\author{
Masatake Nakanishi ${ }^{1}$ and Eizo Kinoshita ${ }^{2}$ \\ 'Nagoya Keizai University \\ Nagaoya, Japan \\ ${ }^{2}$ Meijo University \\ Kani 509-0261, Japan \\ nakanishi-m@nagoya-ku.ac.jp / kinoshit@urban.meijo-u.ac.jp
}

\begin{abstract}
:
This paper proposes an application of a method that bridges between individual and group AHP by using a shared concept : "decision making stress" .

By "Inter-viewpoint stress method", various evaluation data varied by evaluation viewpoints are synthesized with the minimum inter-viewpoint stress. This method grades the viewpoints in such a way to minimize the sum total of differences among each evaluation from each viewpoint, the "Interviewpoint stress," without modifying the raw data of the pairwise comparison. The circulation relations as well as the conflicts between symmetric elements in the pairwise comparison matrix could not be effectively processed by the conventional eigenvector method. The inter-viewpoint stress method, on the contrary, is able to solve these problems. Value of the inter-viewpoint stress is effective as a consistency index for the inter-viewpoint stress method.

"Group decision making stress method" grades the evaluators in such a way to minimize the sum total of each evaluator's frustration, the "group decision making stress," without modifying the raw data of each evaluator's preference. By rationally grading the participants, those who tend to share similar preferences with others would be graded relatively high and those with unique preferences would be graded relatively low. Every preference, however, is appropriately taken into account, and the result shall be fair. Applications of the method will allow an easier search of groups with similar preferences and help to converge the group preference.

As both stress methods apply the same mathematical planning structure for stress minimizing, it is possible to grade each evaluator's viewpoints respectively as to minimize the sum total of each evaluator's frustration in group decision making.
\end{abstract}

